\title{
Antitumor efficacy induced by a B16F10 tumor cell vaccine treated with mitoxantrone alone or in combination with reserpine and verapamil in mice
}

\author{
XIANGFENG HE $^{1 *}$, JING WANG $^{2 *}$, JUN DOU $^{1 *}$, FANGLIU YU $^{1}$, \\ KAI CAI ${ }^{1}$, XIAOLI LI ${ }^{1}$, HONGYI ZHANG ${ }^{1}$ and NING GU ${ }^{3}$ \\ ${ }^{1}$ Department of Pathogenic Biology and Immunology, Medical School; ${ }^{2}$ Department of Gynecology and Obstetrics, \\ Zhongda Hospital; ${ }^{3}$ School of Biological Science and Medical Engineering, Southeast University, Nanjing, P.R. China
}

Received February 8, 2011; Accepted April 20, 2011

DOI: $10.3892 /$ etm.2011.283

\begin{abstract}
An apoptotic tumor cell serves as a potential potent trigger for the initiation of naturally occurring tumor immunity. In the present study, a B16F10 tumor cell vaccine treated with mitoxantrone (MIT) was developed, and its antitumor effect on mice was evaluated. The results indicated that the B16F10 tumor cell vaccine treated with MIT alone or in combination with reserpine (RP) and verapamil (VP) for $12 \mathrm{~h}$ triggered apoptosis, and that the expression of CD80, the MHC II class molecule, NKG2D and its ligand were significantly increased compared to the expression levels in the control group. The tumor vaccine immunogenicity was significantly enhanced in the vaccinated mice, resulting in augmented cytotoxicity of splenocytes and NK cells as well as the splenocyte proliferative response compared to the control group mice. Notably, the mice vaccinated with the B16F10 tumor cell vaccine treated with MIT, RP and VP did not generate tumors only after 60 days into the observation, but the mice also generated a powerful immune prophylactic efficiency against the B16F10 tumor cell challenge. These findings demonstrated the safety and efficacy of the B16F10 tumor cell vaccine treated with MIT alone or in combination with RP and VP in the murine model, and suggest that an apoptotic tumor cell vaccine modeled on naturally occurring tumor immune responses in vivo may provide a safe and immunogenic tumor vaccine for potential applications in humans.
\end{abstract}

\section{Introduction}

Tumor cell vaccines are a promising emerging treatment option to cure tumors, although novel immunotherapeutic options for

Correspondence to: Dr Jun Dou, Department of Pathogenic Biology and Immunology, Medical School, Southeast University, Nanjing 210009, P.R. China

E-mail: njdoujun@yahoo.com.cn

*Contributed equally

Key words: tumor vaccine, mitoxantrone, apoptotic cells, reserpine, verapamil all types of tumors have been recently developed. Apoptotic tumor cells can be recognized and eliminated by the power of the immune response, which has resulted in the intense interest in the development of tumor cell vaccines transfected with DNA containing target genes, immune molecules or treated using different biological methods (e.g., freeze-thawing, large amounts of ultraviolet light, $\gamma$-irradiation or anticancer drugs). These tumor cell vaccines are currently being evaluated as prophylactic and therapeutic vaccines for tumors $(1,2)$.

Mitoxantrone (MIT), a non-cell cycle-specific anthraquinone anticancer drug, induces cell apoptosis by inhibiting DNA synthesis. Treatment of tumor cells with MIT was found to result in translocation of calreticulin from endoplasmic reticulum to the cell surface along with cell apoptosis, which leads to the increased immunogenicity of tumor cells. These MIT-treated apoptotic B16F10 cells may be used as a type of tumor cell vaccine to initiate an effective antitumor immunoresponse in mice (3). The drug-treating tumor cells inoculated into mice are able to be effectively recognized by dendritic cells (DCs) and stimulate the immune system to effectively eliminate tumor cells that are resistant to chemotherapeutic drugs (4). However, certain tumor cells exhibiting a high degree of malignancy, such as murine melanoma B16F10 cells, express ATP-binding cassette (ABC) transporter proteins, such as multidrug resistance 1 (MDR1 or ABCB1), the multidrug resistance protein 1, (MRP1 or ABCC1), ABCB5 and breast cancer resistance protein 1 (ABCG2/BCRP1) (5-7). These multidrug-resistant proteins are able to rapidly 'pump out' anticancer drug from tumor cells, so that a few of the tumor cells that were treated with anticancer drugs survive and generate tumors in vivo. In order to assess the feasibility, safety and immunogenicity of tumor cell vaccines, efforts are ongoing.

Verapamil (VP) and reserpine (RP) are known inhibitors of efflux pumps and block the function of multidrug-resistant proteins, resulting in the retention of anticancer drugs in the tumor cells (8-10). In the present study, B16F10 cells were pretreated with VP and RP to inhibit the activity of the efflux of MIT in the cells, and then B16F10 cells were treated with MIT to cause retention of MIT in the cells. The treated tumor cells gradually underwent apoptosis in the experimental mice and were phagocytosed by autologous DCs. This finally induced 
mice to generate the initiation of naturally occurring antitumor immunity that partly prevented tumor growth. Here, we report the safety and immunogenicity of an apoptotic B16F10 tumor cell vaccine in a controlled study of C57BL/6 mice.

\section{Materials and methods}

Animals and cells. Female C57BL/6 mice, 6-8 weeks of age, 18-20 g, were obtained from the University of Yangzhou, China. All mice were housed under pathogen-free conditions, and the experiments were performed in compliance with the Guidelines of the Animal Research Ethics Board of Southeast University. The B16F10 murine melanoma cell line is syngeneic in C57Bl/6 mice and was a gift from Professor Pingsheng Chen, Medical School, Southeast University; the YAC-1 cell line (Moloney leukemia-induced T-cell lymphoma of A/Sn mouse origin) was obtained from the Cellular Institute of China in Shanghai. These cells were cultured at $37^{\circ} \mathrm{C}$ in a $5 \%$ $\mathrm{CO}_{2}$ atmosphere in RPMI-1640 supplemented with $10 \%$ fetal bovine serum which contained $100 \mathrm{U} / \mathrm{ml}$ penicillin $\mathrm{G}$ sodium and $100 \mu \mathrm{g} / \mathrm{ml}$ streptomycin sulfate.

Animal experiments. In the safety experiment, C57BL/6 mice were first divided into three experimental groups and one control group. Each mouse was subcutaneously (s.c.) immunized in the abdominal region with $2 \times 10^{5}, 5 \times 10^{5}$ or $1 \times 10^{6}$ B16F10 cells treated with MIT for $12 \mathrm{~h}$, or $1 \times 10^{5}$ wildtype B16F10 cells as control. C57BL/6 mice were secondly divided into four experimental groups and one control group. Each mouse was s.c. immunized in the abdominal region with $1 \times 10^{6} \mathrm{~B} 16 \mathrm{~F} 10$ cells treated with MIT in combination with RP and VP for $12 \mathrm{~h}$, or $1 \times 10^{5}$ wild-type B16F10 cells as control.

In the antitumor effect experiment, C57BL/6 mice were randomly divided into the experimental, the B16F10 cell and the PBS control groups. Each mouse was immunized s.c. in the abdominal region, respectively, with a vaccine of $5 \times 10^{5} \mathrm{~B} 16 \mathrm{~F} 10$ tumor cells treated with MIT in combination with RP and VP for $12 \mathrm{~h}$ or $1 \times 10^{5}$ wild-type B16F10 cells inactivated with mitomycin $\mathrm{C}$ or $100 \mu \mathrm{l}$ PBS. The same immunization was repeated twice at an interval of 2 weeks. Two weeks after the second immunization, $1 \times 10^{5} \mathrm{~B} 16 \mathrm{~F} 10$ cells were injected into the abdominal region of each mouse. Tumor growth was monitored twice a week and tumor-free and surviving mice were observed for 60 days. Ten mice/group were used routinely in each experiment $(3,11)$.

B16F10 cells $\left(5 \times 10^{5}\right)$ treated with MIT alone or in combination with RP and VP were inoculated into 24-well plates in RPMI-1640 medium. The drugs were applied at concentrations as follows: $2 \mu \mathrm{g} / \mathrm{ml} \mathrm{MIT,} 2 \mu \mathrm{g} / \mathrm{ml} \mathrm{MIT}+1 \mu \mathrm{g} / \mathrm{ml} \mathrm{RP}$, $2 \mu \mathrm{g} / \mathrm{ml} \mathrm{MIT}+1 \mu \mathrm{g} / \mathrm{ml} \mathrm{VP}$ and $2 \mu \mathrm{g} / \mathrm{ml} \mathrm{MIT} \mathrm{in} \mathrm{combination}$ with $1 \mu \mathrm{g} / \mathrm{ml} \mathrm{RP}$ and $1 \mu \mathrm{g} / \mathrm{ml} \mathrm{VP}$, respectively. The samples at different time points $(0,12,48,96$ and $144 \mathrm{~h})$ were obtained to detect cell apoptosis with the Annexin V-EGFP Apoptosis Detection kit (KeyGen Biotech. Co. Ltd., Nanjing, China). The morphology of apoptotic cells was observed under a fluorescence microscope (TE2000-E fluorescence inverted phase contrast microscope; Nikon Corp.).

Preparation of DCs and analysis of molecular expression. To prepare autologous DCs from mouse bone marrow, the protocol was performed as described previously (12). The collected DCs were used for incubation together with the treated B16F10 cells. For the analysis of molecules of major histocompatibility complex (MHC) class II, cluster of differentiation (CD)80 and CD11c on the DCs, the NKG2D ligand on the B16F10 tumor cell vaccine and NKG2D on the splenocytes, the experiment protocol was performed according to the manufacturer's protocol (eBioscience, USA) (13). Briefly, DCs, the B16F10 tumor cell vaccine and splenocytes were stained with the rabbit anti-mouse MHC class II-PE, CD80-APC and CD11c-FITC, rat anti-mouse NKG2D ligand-PE and rat antimouse NKG2D-FITC monoclonal antibodies (eBioscience), respectively, and subsequent steps were performed according to the protocol provided in the kit (14).

RNA isolation and RT-PCR. The PCR sense primer sequence for the ABCB1 gene was 5'-CGAATGTCTGAGGACAAGCCAC-3' and the anti-sense was 5'-CCATGAGGTCCTGGGCATG-3'. PCR sense primer sequence for the $\beta$-actin gene was 5'-GGACTTCGAGCAAGAGATGG-3' and the anti-sense was 5'-AGCACTGTGTTGGCGTACAG-3'. Total cellular RNA was extracted from $1 \times 10^{6}$ B16F10 cells by using the RNeasy Mini kit (Qiagen, Valencia, CA, USA), according to the manufacturer's instructions. cDNA was synthesized with the reverse Super-Script Choice System (Invitrogen, Carlsbad, CA, USA). cDNAs of ABCB1 and $\beta$-actin were respectively amplified by PCR with the above-mentioned primers (15).

Detection of side population cells in B16F10 cells. B16F10 cells, $70 \%$ confluent in complete medium, were transferred to 6-well plates for $24 \mathrm{~h}$, and Hoechst $33342(5 \mu \mathrm{g} / \mathrm{ml})$ was added to each well and maintained for $1 \mathrm{~min}$ at room temperature. The cells were then washed with PBS three times and subsequently observed under a light and fluorescence microscope, respectively (16).

Effect of RP and VP on the exclusion rate of MIT in B16F10 cells. B16F10 cells $\left(70 \%\right.$ confluent, $\left.1 \times 10^{6}\right)$ in complete medium were transferred to 6-well plates for $24 \mathrm{~h}$ and then $1 \mu \mathrm{g} / \mathrm{ml} \mathrm{RP}$ and $1 \mu \mathrm{g} / \mathrm{ml} \mathrm{VP}$ were added to the wells for $30 \mathrm{~min}$ to inhibit ABCB1 efflux pumps. Then, $2 \mu \mathrm{g} / \mathrm{ml}$ MIT was added to the wells and cultured for another $12 \mathrm{~h}$. Next, the cells were washed with PBS three times and then fixed using $1 \%$ paraformaldehyde. The exclusion rate of MIT was detected by fluorescence confocal microscopy under 488-nm excitation light and 675-nm emission light $(17,18)$.

Assays of splenocyte cytotoxicity and splenocyte proliferative response. Two weeks after the final immunization, 10 mice were sacrificed to detect immune efficiency. Splenocytes $\left(5 \times 10^{6}\right)$ were prepared from the C57BL/6 mice immunized with the $5 \times 10^{5}$ B16F10 tumor cell vaccine treated with MIT in combination with RP and VP. The harvested splenocytes were labeled with $0.5 \mathrm{mM} 5$-(and 6)-carboxy-fluorescein diacetate succinimidyl ester (CFSE; $20 \mu \mathrm{g} / \mathrm{ml}$ ) at $37^{\circ} \mathrm{C}$ for $15 \mathrm{~min}$. After the incubation, the splenocytes were washed twice in PBS containing 5\% fetal bovine serum to sequester any free CFSE that had failed to diffuse into the cells. Murine splenocytes $\left(2 \times 10^{6}\right)$ were resuspended in $10 \%$ RPMI medium in 6-well plates and then incubated with $2 \times 10^{4}$ B16F10 cells inactivated with mitomycin c 
A

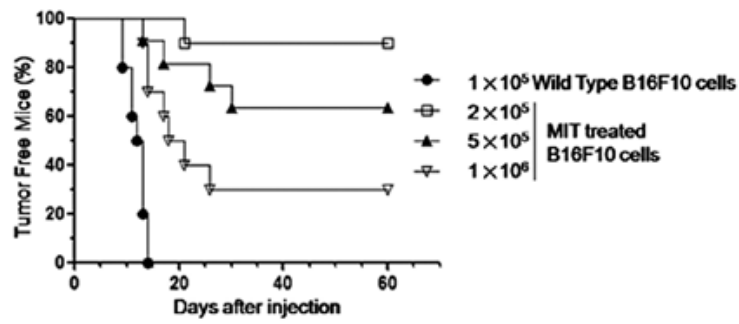

C

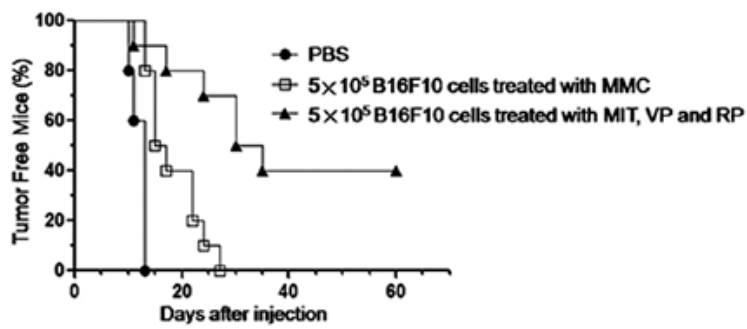

B

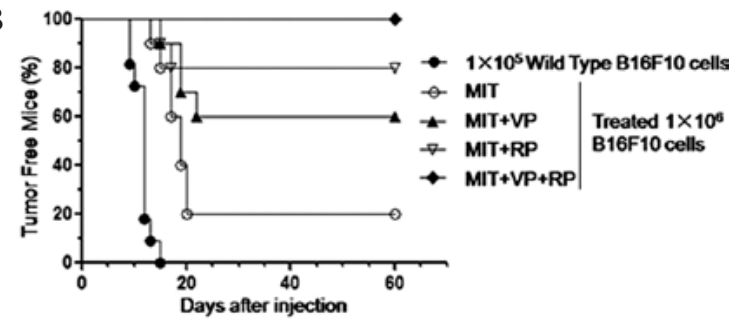

Figure 1. Evaluation of the safety of the B16F10 tumor cell vaccine treated with MIT alone or in combination with VP and RP, as well as its antitumor efficacy. (A) Percentage of tumor-free mice after various injections of MIT-treated B16F10 cells. (B) Percentage of tumor-free mice after injection of B16F10 cells treated with MIT alone or in combination with VP and RP. (C) Mice generated a powerful prophylactic effect against $1 \times 10^{5}$ B16F10 cell challenge after being immunized with the $5 \times 10^{5}$ B16F10 tumor cell vaccine treated with MIT, VP and RP.

$(100 \mu \mathrm{g} / \mathrm{ml})$ for $72 \mathrm{~h}$. The cells were then rinsed extensively with complete medium and used in the proliferative assay $(19,20)$.

ELISA for IFN- $\gamma$. The serum IFN- $\gamma$ level was measured using a commercially available enzyme linked-immunosorbent assay according to the manufacturer's protocol (eBioscience) $(2,11)$.

Statistical analysis. For each group of mice, data were represented as the mean value of each group and its associated standard deviation. The Student's t-test for two-group comparison and Bonferroni correction for multiple comparisons were used to determine significant differences. $\mathrm{p}<0.05$ was considered statistically significant.

\section{Results}

Safety of the B16F10 tumor cell vaccine and its antitumor efficacy in mice. In the present study, we first assessed the safety of the B16F10 tumor cell vaccine treated with MIT. After the B16F10 cells were treated with $2 \mu \mathrm{g} / \mathrm{ml}$ MIT for $12 \mathrm{~h}$, the cells began to undergo apoptosis and were then injected into the mice (10 mice/group). Measurable tumors were detected in the mice injected with the MIT-treated $1 \times 10^{6}$ or $5 \times 10^{5} \mathrm{~B} 16 \mathrm{~F} 10$ cells on day 15 or on day 20 (in the $2 \times 10^{5}$ B16F10 cell group) or on day 10 (in the $1 \times 10^{5}$ wild-type B16F10 cell group), respectively. From the tumorigenesis data, 7 out of 10 mice inoculated with the MIT-treated $1 \times 10^{6}$ tumor vaccine, 4 out of 10 mice inoculated with the MIT-treated $5 \times 10^{5}$ tumor vaccine, and 1 out of 10 mice inoculated with MIT-treated $2 \times 10^{5}$ B16F10 cells, respectively, formed tumors up until 60 days into the observation. The results indicated that the MIT-treated $2 \times 10^{5} \mathrm{~B} 16 \mathrm{~F} 10$ cells still possessed tumorigenic potential in $\mathrm{C} 57 \mathrm{BL} / 6$ mice in spite of only 1 out of 10 mice developing tumors (Fig. 1A). In the subsequent safety experiment, the B16F10 cells were pretreated with $1 \mu \mathrm{g} / \mathrm{ml} \mathrm{RP}$ or/and $1 \mu \mathrm{g} / \mathrm{ml} \mathrm{VP}$ and then the cells were re-treated with $2 \mu \mathrm{g} / \mathrm{ml}$ MIT. The B16F10 cell vaccine treated with MIT $+\mathrm{RP}+\mathrm{VP}$ was complete safety as all 10 mice did not develop tumors until 60 days into the observation. However, in regards to the mice treated with MIT + RP or MIT + VP, 2 out of 10 or
4 out of 10 mice, respectively, still developed tumors (Fig. 1B). Next, we evaluated the antitumor efficacy in mice inoculated with the preparation of $5 \times 10^{5}$ B16F10 cells treated with MIT in combination with RP and VP as a tumor cell vaccine. Fig. $1 \mathrm{C}$ shows that 6 out of 10 mice immunized with the treated $1 \times 10^{6}$ B16F10 tumor cell vaccine twice and challenged by $1 \times 10^{5}$ B16F10 cells formed tumors on days 12, 16, 25, 30, 30 and 35 . The remaining 4 mice did not form tumors throughout the 60-day observation. However, all 10 mice formed tumors in less than 14 days when immunized with PBS or in less than 27 days when immunized with $5 \times 10^{5}$ B16F10 cells inactivated with mitomycin $\mathrm{C}$ twice and challenged by $1 \times 10^{5}$ B16F10 cells. These findings demonstrate that the B16F10 tumor cell vaccine treated with MIT in combination with RP and VP is not only safe, but induces mice to generate a powerful and efficacious antitumor immune response.

Detection of the ABCB1 gene, side population cells and exclusion rate of MIT in B16F10 cells. From the tumorigenesis data, we found that the MIT-treated B16F10 cells still exhibited tumorigenicity in mice. The main reasons may be that the B16F10 cells are malignant cells and have the ability to discharge MIT from the treated B16F10 cells by ABC transporter proteins. Thus, a few of the B16F10 cells did not undergo apoptosis after the MIT treatment. Since ABCB1 is one of the $\mathrm{ABC}$ transporter proteins, we aimed to ascertain whether the ABCB1 gene was present in the B16F10 cells. The RT-PCR result demonstrated expression of the ABCB1 gene (Fig. 3A). It is also known that side population (SP) cells, also termed 'dull cells', usually represent only a small fraction of the whole cell population that are identified by efflux of Hoechst dye through $\mathrm{ABC}$ transporter proteins, and are present in virtually all malignant cells and a part of normal tissues $(21,22)$. Thus, we further detected SP cells in the B16F10 cells. A small fraction of SP cells (Fig. 2B, arrows) existed in the B16F10 cells, nevertheless, the SP cells were invisible in the B16F10 cells after the cells were treated with RP and the VP (Fig. 2C). This was due to the blockage of efflux pumps of ABCB1 by RP and the VP. As a result, the SP cells were not observed under a fluorescence 
A

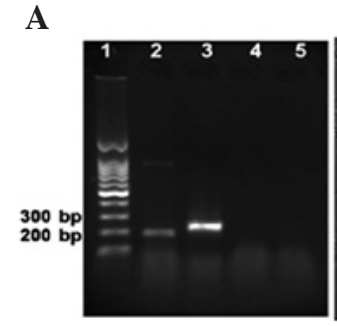

B

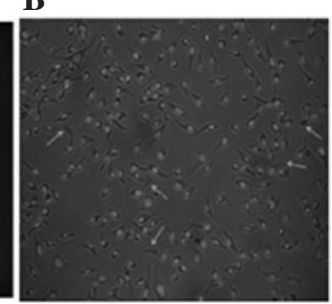

C

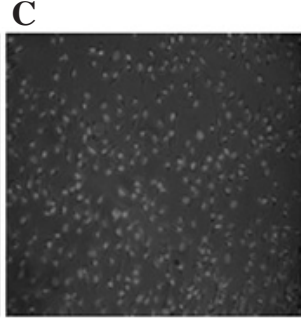

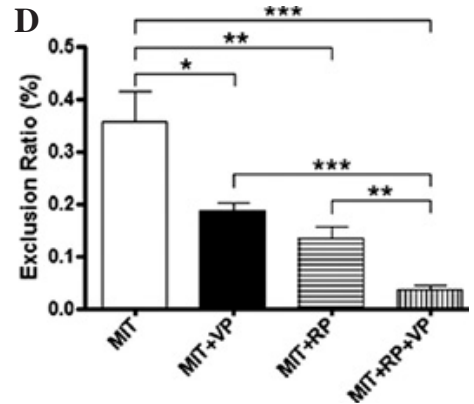

Figure 2. Analysis of ABCB1 gene expression, SP cells and exclusion ratio of MIT in B16F10 cells. (A) Transcriptive expression of the ABCB1 gene (lane 2) and DNA marker and $\beta$-actin (lanes 1 and 3). (B) SP cells (indicated by arrows) were noted in A and B, but were invisible in the same cells in (C). (D) Exclusion ratio in the B16F10 cells treated with MIT alone or in combination with VP and RP, respectively. Statistically significant differences between two groups as judged by the Student's t-test and corrected by Bonferroni analysis; ${ }^{* * *} \mathrm{p}<0.003,{ }^{* *} \mathrm{p}<0.03,{ }^{*} \mathrm{p}<0.05$.

microscope since the Hoechst 33342 stain was retained in the B16F10 cells, which made the SP cells were stained and did not be 'dull cells'. To further assess the function of the inhibition of ABCB1 efflux pumps by RP and the VP, we subsequently assessed the MIT exclusion ratio in the B16F10 cells. As shown in Fig. 2D, the ability to exclude MIT was statistically significantly decreased when the B16F10 cells were concurrently treated with MIT, VP and RP in contrast to the ability of MIT-treated $(\mathrm{p}<0.003)$, MIT + VP-treated $(\mathrm{p}<0.03)$ and MIT + RP-treated B16F10 cells $(\mathrm{p}<0.05)$. The results suggest that RP and VP block the function of ABCB1 efflux of MIT and assist MIT in further inducing B16F10 cell apoptosis.

Detection of apoptotic B16F10 cells treated with MIT in combination with RP and VP as well as molecular expression. Fig. 3A shows that B16F10 cells gradually experienced apoptosis $48 \mathrm{~h}$ after treatment with MIT in combination with RP and VP. In viable apoptotic cells, the cell membrane began to shrink and became a green color. In non-viable apoptotic cells (after $96 \mathrm{~h}$ ), the cell nucleus became a red color, while the cell membrane remained green. When apoptotic B16F10 cells were cultured with the autologous DCs from mouse bone marrow for $72 \mathrm{~h}$, the DCs ingested more apoptotic bodies (Fig. 3B) compared to the control DCs (Fig. 3C). In order to visualize the state of phagocytic apoptotic bodies, DiI-labeled apoptotic B16F10 cells were incubated with the DCs stained for FITC-conjugated CD11c. It was found that the apoptotic B16F10 cells (red) were detected inside DCs (green) by fluorescence microscope analysis (Fig. 3D). As the capability of phagocytic apoptotic B16F10 cells was increased, the molecular expression of CD80 (Fig. 3E) and MHC class II (Fig. 3F) was statistically significantly increased on the surface of DCs, respectively, compared to that of DCs incubated with wild-type B16F10 cells ( $p<0.05$ or $<0.01)$. The increase in the molecular expression suggested that DCs underwent a maturation process and served as a potential trigger for the initiation of a immune response in vivo.

In addition, the expression of the $\mathrm{NKG} 2 \mathrm{D}$ ligand in MIT-treated apoptotic B16F10 cells (Fig. 3F) and the expression of $\mathrm{NKG} 2 \mathrm{D}$ in the $\mathrm{NK} 1.1^{+}$cells from the vaccinated mice (Fig. 3G) were statistically significantly increased compared to those of wild-type B16F10 cells, respectively. This data demonstrated that the apoptotic B16F10 tumor cells promoted DC maturation as well as enhanced the expression of NKG2D and its ligand.
Immune efficacy induced by the B16F10 tumor cell vaccine treated with MIT in combination with RP and VP. As shown in Fig. 1C, the B16F10 tumor cell vaccine treated with MIT in combination with RP and VP induced the mice to elicit a powerful prophylactic efficiency against B16F10 cell challenge. To investigate the main mechanism of the antitumor effect induced by the B16F10 tumor cell vaccine, we assessed the cytotoxicity of splenocytes and NK cells and detected the splenocyte proliferative response and the serum level of IFN- $\gamma$. Data showed that the cytotoxic activity of splenocytes and NK cells was statistically significantly increased $(\mathrm{p}<0.01$ or $<0.05)$, respectively, compared to the control mice (Fig. 4A). This was also verified by the splenocyte proliferative response and the serum level of IFN- $\gamma$ (Fig. 4B and C). The results of the immunological experiment suggest that the B16F10 tumor cell vaccine enhanced the cellular immune function in vaccinated mice, which may be a major mechanism of the antitumor efficacy induced by the B16F10 tumor cell vaccine treated with MIT + RP and VP.

\section{Discussion}

Several studies have revealed that the use of apoptotic cells in vaccines may serve as a potent source of antigens for stimulating host immune responses in vivo $(3,23,24)$. In the present study, the anthracycline drug MIT was used to induce B16F10 cell apoptosis that was immunized into mice to assess the safety of a vaccine of MIT-treated tumor cells. However, the result was not satisfactory as these treated tumor cells retained tumorigenic potential in the $\mathrm{C} 57 \mathrm{BL} / 6$ murine model (Fig. 1A).

The goal of tumor vaccine development is its safeness and effectiveness. Therefore, the reasons why MIT-treated tumor cells possess tumorigenic potential were investigated. The ABCB1 transporter protein (Fig. 2A) and a few SP cells (Fig. 2B) were found in the B16F10 cells. Approximately $0.3 \%$ MIT was able to be discarded from the MIT-treated tumor cells through SP cells via the ABCB1 transporter protein. Thus, a few B16F10 cells did not undergo the apoptotic process and retained tumorigenic potential in vivo. For this reasons, the B16F10 cells were pretreated with RP and VP to block the function of efflux pumps, and then were retreated with MIT. As a result, SP cells disappeared in the B16F10 cells (Fig. 2C), MIT was almost completely maintained in the treated tumor cells (Fig. 2D) and there was nearly complete apoptosis at $144 \mathrm{~h}$ (Fig. 3A). The apoptotic B16F10 cells were utilized as 
A

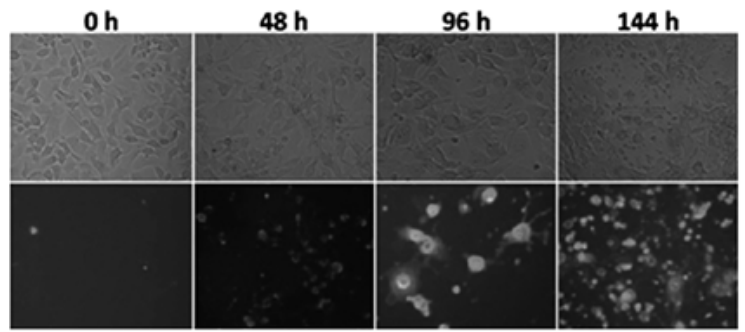

B

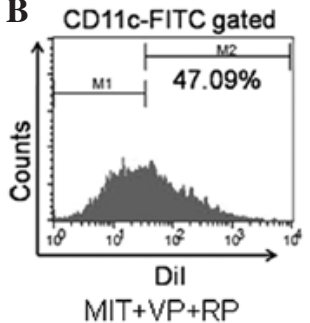

C CD11C-FITC gated

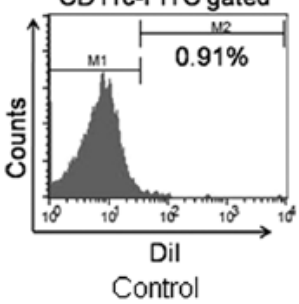

D
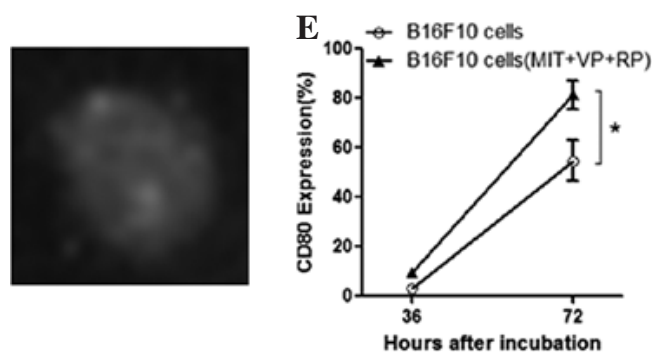

$\mathbf{H}$

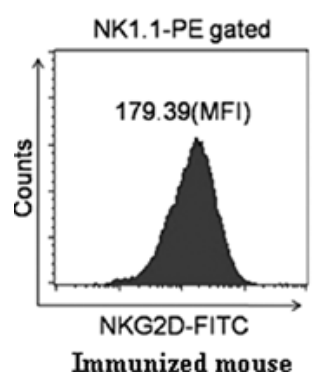

I

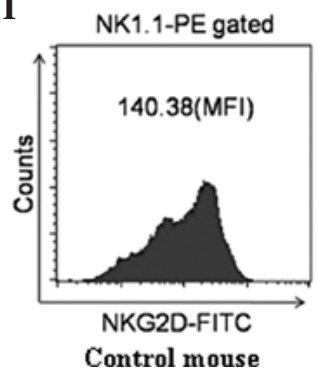

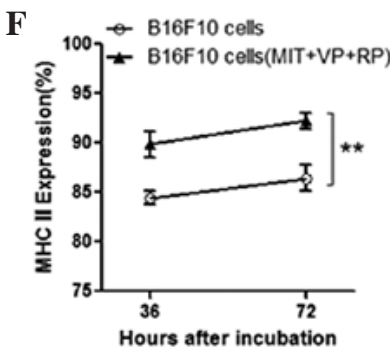

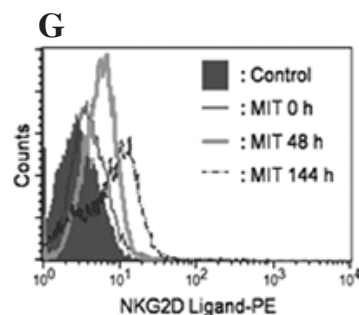

Figure 3. Assays for B16F10 cell apoptosis and detection of DC phagocytosis as well as analysis of molecular expression. (A) Top panels (under a microscope) and bottom panels (under a fluorescence microscope) show B16F10 cells treated with MIT in combination with RP and VP for the different times (h). The phagocytosed apoptotic bodies by DCs were markedly enhanced ( $<<0.001)$ in (B) the B16F10 cells treated with MIT, VP and RP compared to (C) the untreated B16F10 cells. (D) The apoptotic cells (red) were ingested by DC (green) under fluorescence microscope. DCs were incubated with B16F10 cells or MIT+VP+RPtreated B16F10 cells for $72 \mathrm{~h}$ and these cells were stained for the various antibodies to analyze the molecular expression of (E) CD80 and (F) MHC class II by flow cytometry. (G) Expression of the NKG2D ligand was significantly increased in the B16F10 cells treated with MIT for $144 \mathrm{~h}$ compared to that of the control B16F10 cells. Expression of NKG2D (MFI) was significantly increased in NK cells in $(\mathrm{H})$ the mice immunized with the treated tumor cell vaccine $(\mathrm{p}<0.05)$ in contrast to that of (I) the control cells. One representative experiment was repeated three times. MFI, mean fluorescence intensity. ${ }^{* * *} \mathrm{p}<0.001,{ }^{* * *} \mathrm{p}<0.01,{ }^{*} \mathrm{p}<0.05$.

A

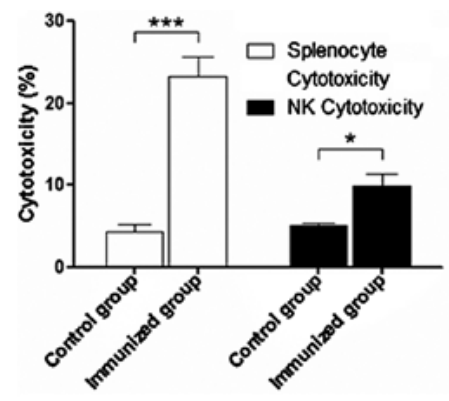

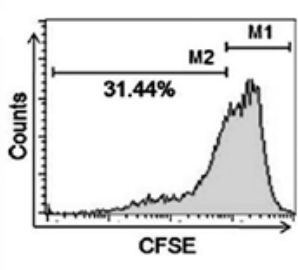

Immunized mouse

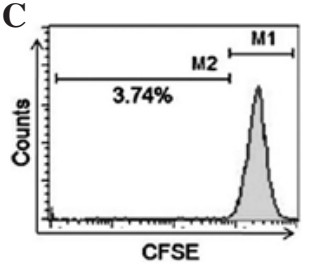

Control mouse

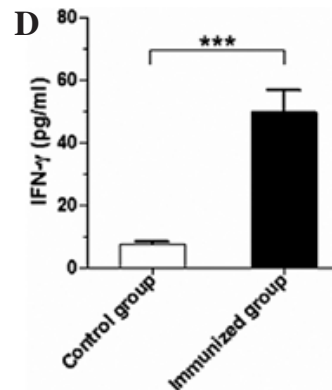

Figure 4. Detection of immune efficacy in the vaccinated mice. (A) Cytotoxicity of splenocytes and NK cells, respectively. The splenocyte proliferative response was significantly increased in (B) the mice immunized with the treated tumor cell vaccine $(\mathrm{p}<0.001)$ in contrast to that of the $(\mathrm{C})$ control cells. (D) Serum IFN- $\gamma$ level. One representative experiment represents 10 individual mice. ${ }^{* * *} \mathrm{p}<0.001,{ }^{*} \mathrm{p}<0.05$.

a tumor vaccine to vaccinate the mice twice and then $\mathrm{B} 16 \mathrm{~F} 10$ cell challenge. The B16F10 tumor cell vaccine treated with MIT in combination with RP and VP was not only completely safe (Fig. 1B), but induced an obvious prophylactic effect against B16F10 cell attack in the murine model (Fig. 1C).

Subsequently, the possible mechanism of antitumor efficacy induced by the preparation of the B16F10 tumor cell vaccine in mice was investigated. It is known that B16F10 tumor cells exhibit low immunogenicity and do not easily elicit an antitumor immune response in murine models. However, apoptotic B16F10 tumor cells induced a strong immune response in the tumor-bearing mice $(3,25)$. For this reason, we induced B16F10 cell apoptosis by using the anthraquinone anticancer drug MIT, in combination with RP and VP (Fig. 3A). When the autologous DCs from mouse bone marrow were concurrently incubated with the apoptotic B16F10 cells for 3 days, the immature DCs developed into mature DCs, resulting in the increased molecular expression of CD80 and MHC class II on the cell surface of DCs, as well as enhancing the ability of phagocytic and present apoptotic B16F10 cells (Fig. 3B-D). DCs capture apoptotic tumor cells, process them and present the relevant antigen 
epitope in the context of both class II and I MHC to prime lymphocytes, which are specialized functions of DCs $(25,26)$. Therefore, the apoptotic B16F10 cells may act as a tumor cell vaccine that may elicit lymphocyte activation via the abovementioned mature DCs in vivo. In addition, the NKG2D receptor (Fig. 3H) and NKG2D ligand (Fig. 3G) were highly expressed in the NK and apoptotic tumor cells, respectively. The NKG2D immunoreceptor interacting with the NKG2D ligand serves as one of the most potent activating receptor and ligand for effector NK cells, playing an important role in the immunosurveillance of tumors (27). Although we did not detect the translocation of calreticulin from endoplasmic reticulum to the cell surface, this mechanism has been confirmed by other researchers $(3,4,28,29)$. Accordingly, we assumed that the treatment of a tumor cell vaccine with MIT would cause cell apoptosis resulting in a calreticulin coating on the surface of apoptotic cells for recognition and uptake by DCs. The presented apoptotic cells by DCs may include a predominant antigen for eliciting lymphocytes in immunized mice to generate strong immune responses $(23,30)$. Consequently, the cytotoxicity of splenocytes and NK cells as well as the splenocyte proliferative response and the serum IFN- $\gamma$ level were markedly enhanced compared to the control mouse group (Fig. 4).

In conclusion, this study demonstrated that the B16F10 tumor cell vaccine treated with MIP in combination with RP and VP was safe and efficient. The apoptotic tumor cells facilitated DC maturation, leading to the activation of lymphocytes in the vaccinated mice. The immune efficacy induced by the treated tumor cell vaccine exhibited powerful prevention against B16F10 cell challenge in the murine model. These data provide knowledge that may be useful for developing an effective B16F10 tumor cell vaccine for the treatment of melanoma patients in clinical trials.

\section{Acknowledgements}

The authors wish to extend gratitude to Dr Lili Chu and Dr Fengshu Zhao for the expert administrative assistance. This study was partly supported by the National Natural Science Foundation of China (no. 81071769) and in part by the 973 Program of China (no. 2011CB933500).

\section{References}

1. Saito H, Frleta D, Dubsky P and Palucka AK: Dendritic cellbased vaccination against cancer. Hematol Oncol Clin North Am 20: 689-710, 2006.

2. Dou J, Chu LL, Zhao FS, et al: Study of immunotherapy of murine myeloma by an IL-21-based tumor vaccine in Balb/c mice. Cancer Biol Ther 6: 1871-1879, 2007.

3. Chunyu C, Yu Han, Yushan R and Yanlin W: Mitoxantronemediated apoptotic B16-F1 cells induce specific anti-tumor immune response. Cell Mol Immunol 6: 469-475, 2009.

4. Obeid M, Tesniere A, Ghiringhelli F, et al: Calreticulin exposure dictates the immunogenicity of cancer cell death. Nat Med 13 54-61, 2007.

5. Mimeault M, Hauke R, Mehta PP, et al: Recent advances in cancer stem/progenitor cell research: therapeutic implications for overcoming resistance to the most aggressive cancers. J Cell Mol Med 11: 981-1011, 2007.

6. An Y and Ongkeko WM: ABCG2: the key to chemoresistance in cancer stem cells? Expert Opin Drug Metab Toxicol 5: 1529-1542, 2009.

7. Schinkel AH and Jonker JW: Mammalian drug efflux transporters of the ATP binding cassette (ABC) family: an overview. Advanced Drug Delivery Rev 55: 3-29, 2003.
8. Van Veen HW, Venema K, Bolhuis H, et al: Multidrug resistance mediated by a bacterial homolog of the human multidrug transporter MDR1. Proc Natl Acad Sci USA 93: 10668-10672, 1996.

9. Droi S, Eytan GD and Assaraf YG: Potentiation of anticancerdrug cytotoxicity by multidrug-resistance chemosensitizers involves alterations in membrane fluidity leading to increased membrane permeability. Eur J Biochem 228: 1020-1029, 1995.

10. Spengler G, Viveiros M, Martins M, et al: Demonstration of the activity of P-glycoprotein by a semi-automated fluorometric method. Anticancer Res 29: 2173-2177, 2009.

11. Fengshu Z, Jun D, XiangFeng $\mathrm{H}$, et al: Enhancing therapy of B16F10 melanoma efficacy through tumor vaccine expressing GPI-anchored IL-21 and secreting GM-CSF in mouse model. Vaccine 28: 2846-2852, 2010.

12. Hyesung K, Hyemi P, Jungsun P, et al: Dendritic cell vaccine in addition to FOLFIRI regimen improve antitumor effects through the inhibition of immunosuppressive cells in murine colorectal cancer model. Vaccine 28: 7787-7796, 2010.

13. Groh V, Steinle A, Spies T, et al: Recognition of stress-induced MHC molecules by intestinal epithelial gammadelta $T$ cells. Science 279: 1737-1740, 1998.

14. Jun D, Yongfang, W, Jing W, et al: Antitumor efficacy induced by human ovarian cancer cells secreting IL-21 alone or combination with GM-CSF cytokines in nude mice model. Immunobiology 214: 483-492, 2009.

15. Jun D, Quan T, Fengshu Z, et al: Comparison of immune responses induced in mice by vaccination with DNA vaccine constructs expressing mycobacterial antigen 85A \& interleukin-21 and Bacillus Galmette-Guérin. Immunol Invest 37: 113-127, 2008.

16. Dou J, Wen $\mathrm{P}, \mathrm{Hu} \mathrm{W}$, et al: Identifying tumor stem-like cells in mouse melanoma cell lines by analyzing the characteristics of side population cells. Cell Biol Int 33: 807-815, 2009.

17. Karla PK, Earla R, Boddu SH, et al: Molecular expression and functional evidence of a drug efflux pump (BCRP) in human corneal epithelial cells. Curr Eye Res 34: 1-9, 2009.

18. Yuan JH, He ZM, Yu YH, et al: Expression establishment and functional analysis of breast cancer resistance protein with doxycycline induced tet regulating system in mouse fibroblast cell line PA317. Ai Zheng 23: 1127-1133, 2004.

19. Lecoeur H, Février M, Garcia S, Rivière Y, Gougeon ML: A novel flow cytometric assay for quantitation and multiparametric characterization of cell-mediated cytotoxicity. J Immunol Methods 253: 177-187, 2001.

20. Fengshu Z, Jun D, Wang J, et al: Investigation on the anti-tumor efficacy by expression of GPI-anchored mIL-21 on the surface of B16F10 cells in C57BL/6 mice. Immunobiology 215: 89-100, 2010.

21. Goodell MA, Brose K, Paradis G, et al: Isolation and functional properties of murine hematopoietic stem cells that are replicating in vivo. J Exp Med 183: 1797-1806, 1996.

22. Jun D and Ning G: Emerging strategies for the identification and targeting of cancer stem cells. Tumor Bio 31: 243-253, 2010.

23. Frank MO, Kaufman J, Tian S, et al: Harnessing naturally occurring tumor immunity: a clinical vaccine trial in prostate cancer. PLoS One 5: e12367, 2010.

24. Taniguchi K, Nishiura H, Ota Y, et al: Roles of the ribosomal protein S19 dimer and chemically induced apoptotic cells as a tumor vaccine in syngeneic mouse transplantation models. J Immunother 34: 16-27, 2011.

25. Banchereau J and Steinman R: Dendritic cells and the control of immunity. Nature 392: 245-152, 1998.

26. Gardai SJ, McPhillips KA, Frasch SC, et al: Cell-surface calreticulin initiates clearance of viable or apoptotic cells through trans-activation of LRP on the phagocyte. Cell 123: 321-334, 2005.

27. Hu W, Wang J, Dou J, et al: Augmenting therapy of ovarian cancer efficacy by secreting IL-21 human umbilical cord blood stem cells in nude mice. Cell Transplant: Nov. 5, 2010 (E-pub ahead of print)

28. Panaretakis T, Joza N, Modjtahedi N, et al: The co-translocation of ERp57 and calreticulin determines the immunogenicity of cell death. Cell Death Differ 15: 1499-1509, 2008.

29. Davide B, Stefano G, Giovanna C, et al: Post-apoptotic tumors are more palatable to dendritic cells and enhance their antigen cross-presentation activity. Vaccine 26: 6422-6432, 2008.

30. Palucka AK, Ueno H, Fay JW, et al: Taming cancer by inducing immunity via dendritic cells. Immunol Rev 220: 129-150, 2007. 